\title{
Swift UVOT near-UV transit observations of WASP-121 $b^{\star}$
}

\author{
M. Salz ${ }^{1}$, P. C. Schneider ${ }^{1}$, L. Fossati ${ }^{2,3}$, S. Czesla $^{1}$, K. France ${ }^{4}$, and J. H. M. M. Schmitt ${ }^{1}$ \\ ${ }^{1}$ Hamburger Sternwarte, Universität Hamburg, Gojenbergsweg 112, 21029 Hamburg, Germany \\ e-mail: msalz@hs.uni-hamburg.de \\ ${ }^{2}$ Space Research Institute, Austrian Academy of Sciences, Schmiedlstrasse 6, 8042 Graz, Austria \\ ${ }^{3}$ School of Physical Sciences, The Open University, Walton Hall, Milton Keynes MK7 6AA, UK \\ ${ }^{4}$ Laboratory for Atmospheric and Space Physics, University of Colorado, 600 UCB, Boulder, CO 80309, USA
}

Received 5 December 2017 / Accepted 23 January 2019

\begin{abstract}
Close-in gas planets are subject to continuous photoevaporation that can erode their volatile envelopes. Today, ongoing mass loss has been confirmed in a few individual systems via transit observations in the ultraviolet spectral range. We demonstrate that the Ultraviolet/Optical Telescope (UVOT) onboard the Neil Gehrels Swift Observatory enables photometry to a relative accuracy of about $0.5 \%$ and present the first near-UV (200-270 nm, NUV) transit observations of WASP-121 b, a hot Jupiter with one of the highest predicted mass-loss rates. The data cover the orbital phases $0.85-1.15$ with three visits. We measure a broadband NUV transit depth of $2.10 \pm 0.29 \%$. While still consistent with the optical value of $1.55 \%$, the NUV data indicate excess absorption of $0.55 \%$ at a $1.9 \sigma$ level. Such excess absorption is known from the WASP-12 system, and both of these hot Jupiters are expected to undergo mass loss at extremely high rates. With a Cloudy simulation, we show that absorption lines of Fe II in a dense extended atmosphere can cause broadband near-UV absorption at the $0.5 \%$ level. Given the numerous lines of low-ionization metals, the NUV range is a promising tracer of photoevaporation in the hottest gas planets.
\end{abstract}

Key words. planets and satellites: atmospheres - techniques: photometric - planets and satellites: physical evolution planet-star interactions - planets and satellites: individual: WASP-121 b - planets and satellites: individual: WASP-12 b

\section{Introduction}

Shortly after its discovery (Henry et al. 2000; Charbonneau et al. 2000), the first transiting hot Jupiter HD 209458 b was found to host an extended hydrogen atmosphere that covers approximately $15 \%$ of the host star during transit (Vidal-Madjar et al. 2003). The atmospheric expansion is thought to be caused by the absorption of high-energy stellar photons (X-rays and extreme UV, XUV, $\lambda<912 \AA$ ). The ensuing heat input raises the temperature in planetary thermospheric layers to about $10^{4} \mathrm{~K}$ and accelerates atmospheric gas to supersonic speeds. This results in the formation of a planetary wind that somewhat resembles the solar wind (Parker 1958). Estimates for the resulting planetary mass-loss rates are often obtained by assuming that this planetary wind is energy-limited (Watson et al. 1981). However, the true nature of such winds can be more complex, because simulations have shown a transition from the energyto a recombination-limited regime (Owen \& Alvarez 2016; Salz et al. 2016a). Additionally, interactions with the stellar wind or a planetary magnetic field further complicate matters (e.g., Murray-Clay et al. 2009; Khodachenko et al. 2015).

Planetary winds can be studied indirectly, because they are thought to shape the radius distribution of short-period planets (Lundkvist et al. 2016; Fulton et al. 2017). However, direct observations of evaporating atmospheres in four systems have been crucial for our understanding of atmospheric escape: HD 209458 b, HD 189733 b, WASP-12 b, and GJ 436 b

* Light curves shown in Fig. A.1 are available at the CDS via anonymous ftp to cdsarc.u-strasbg.fr (130.79.128.5) or via http://cdsarc.u-strasbg.fr/viz-bin/qcat?J/A+A/623/A57
(Vidal-Madjar et al. 2003; Lecavelier des Etangs et al. 2010, 2012; Linsky et al. 2010; Fossati et al. 2010; Haswell et al. 2012; Kulow et al. 2014; Ehrenreich et al. 2015; Lavie et al. 2017).

Among the direct detections, WASP-12 is the most extreme system: the planet orbits just above the Roche-lobe limit and experiences one of the highest irradiation levels of any hot Jupiter (Hebb et al. 2009). This results in a powerful planetary wind, as evidenced by Hubble Space Telescope (HST) Cosmic Origins Spectrograph (COS) observations that have revealed a transit depth of 3\% in three near-UV (NUV) bands between 254 and $283 \mathrm{~nm}$. The NUV transit is about two times deeper than the optical one of 1.4\% (Fossati et al. 2010; Haswell et al. 2012; Nichols et al. 2015). This planet is likely surrounded by a thick, escaping atmosphere in which metals are dragged into the upper atmosphere and low-ionization metallic absorption lines cause the additional broadband NUV absorption (Fossati et al. 2010).

\section{The WASP-121 system}

With respect to the expected planetary mass loss, WASP-121 is a close analog to the WASP-12 system (Delrez et al. 2016), but the host star is four times brighter. A hot Jupiter orbits the bright F6-type main-sequence star $\left(V=10.4, T_{\text {eff }}=6460 \mathrm{~K}\right)$ in 1.27 days, close to the tidal disruption limit $(1.15 \times$ Roche limit, Delrez et al. 2016). WASP-121 b is subject to an exceptionally high bolometric insolation of $7.1 \times 10^{9} \mathrm{erg} \mathrm{cm}^{-2} \mathrm{~s}^{-1}, 5200$ times that of the Earth. It exhibits a mean density of only $0.183 \mathrm{~g} \mathrm{~cm}^{-3}$ and in Sect. 7 we further derive a high level of XUV irradiation. This should lead to severe mass loss, since the evaporation 
mainly depends on the irradiation level and the mean planetary density (e.g., Erkaev et al. 2007).

Activity of the host star manifests itself in an elevated level of radial-velocity (RV) jitter, which has provided an estimate for the stellar rotation period of $\approx 1 \mathrm{~d}$ (Delrez et al. 2016). The star is likely seen at a low inclination about $10^{\circ}$ with the planet in a nearly polar orbit. The lack of $\mathrm{Ca}$ II $\mathrm{H}$ and $\mathrm{K}$ emission line cores in a presumably active star is reminiscent of the WASP-12 system, where material evaporated from the hot Jupiter is thought to veil stellar chromospheric emission (Fossati et al. 2013).

HST Wide Field Camera 3 observations of the primary and secondary transits have revealed $\mathrm{H}_{2} \mathrm{O}$ and possibly indicate TiO, $\mathrm{VO}$, and $\mathrm{FeH}$ in the planetary atmosphere (Evans et al. 2016; Tsiaras et al. 2018). $\mathrm{H}_{2} \mathrm{O}$ emission in the secondary transit provides the first clear evidence of a stratospheric temperature inversion in a hot Jupiter (Evans et al. 2017).

\section{Photometric stability of Swift's UVOT}

Ultraviolet transit observations with the HST have revealed four examples of escaping atmospheres, but HST resources are limited. The Ultraviolet/Optical Telescope (UVOT) onboard the Neil Gehrels Swift Observatory also provides an opportunity to obtain space-based UV photometry, albeit with a smaller telescope (30 cm primary mirror compared to HST's $2.4 \mathrm{~m}$ mirror, Roming et al. 2005; Poole et al. 2008; Breeveld et al. 2010). The instrument is a micro-channel plate intensified photon counting detector. In event mode each photon is registered with the timing resolution of the CCD read out (full frame $\approx 11 \mathrm{~ms}$ ). Swift is in a low Earth orbit with a period of $85 \mathrm{~min}$, allowing for undisturbed visibility windows of typically about $30 \mathrm{~min}$ - a little shorter than the visibility windows of the HST. Hence, transit observations that last a few hours must extend over several spacecraft orbits.

To test the stability of the UVOT and determine its suitability for transit observations, we analyzed an archival data set of 55 Cnce (observation IDs 00034849001-00034849012). The host star was observed in three visits over four consecutive spacecraft orbits and in one visit over five orbits (24 Dec. 2016, 18 Mar. 2017, 19 Mar. 2017, and 12 May 2017). The chosen $u v m 2$ filter has a central wavelength of $225 \mathrm{~nm}$ and a full width at half maximum (FWHM) of $50 \mathrm{~nm}$. To achieve the best photometric accuracy, the target was placed onto similar positions on the detector, which requires two correction slews. Thus, prior to the about $1500 \mathrm{~s}$ long science exposure, each orbit contains two acquisition exposures of about $200 \mathrm{~s}$ duration. The final light curves are affected by known regions of low sensitivity, which are likely caused by dust depositions on the photocathode. Since no corrections are available, we excluded two affected exposures (see the procedure in the appendix and SWIFT-UVOT-CALDB-17-01 $b^{1}$ ).

We reduced the data using HEASOFT (v6.20). The tasks coordinator and uvotscreen were run with standard input parameters to obtain an event table in sky coordinates, filtered for good time intervals and data quality. The attitude control during these observations was inaccurate, which led to a rejection of large chunks of data unless photons with the imperfect attitude flag 256 were accepted (P. Kuin, priv. comm.).

To account for the residual motion of the source caused by the imperfect attitude control, we devised a nonstandard reduction procedure in collaboration with the UVOT team to increase the photometric stability. We used uvotscreen to slice

\footnotetext{
1 https://heasarc.gsfc.nasa.gov/docs/heasarc/caldb/ swift/docs/uvot/
}

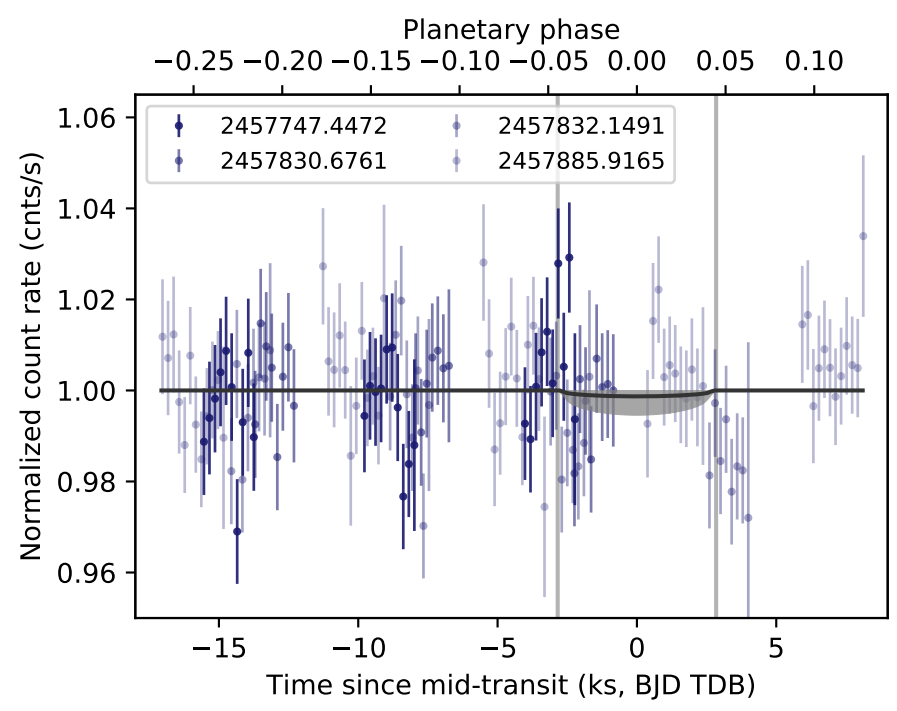

Fig. 1. Swift UVOT transit light curves of $55 \mathrm{Cnc}$ e binned to $200 \mathrm{~s}$. Individual visits are color coded; mid-transit times are given in the legend. The planetary transit is marked by vertical lines. The best fit MandelAgol transit model (black line) is shown with the $95 \%$ confidence region (gray shaded area): a planetary transit is not detected. The four light curves visualize the stability of Swift's UVOT.

the event tables into $20 \mathrm{~s}$ bins, determined the source positions in each slice via iteratively finding its centroid in ds9, and then ran the standard uvotevtlc routine on each slice to apply the corrections for background, aperture, coincidence loss, dead time, large-scale sensitivity variations, and long-term sensitivity variations. The light curve slices were merged with ftmerge and the time stamps were converted to the barycentric dynamical time (TDB) scale via barycorr.

The obtained light curves of $55 \mathrm{Cnc}$ are shown in Fig. 1. The hot super-Earth has a visual transit depth of $0.04 \%$, which is not detected in the UVOT data. With a count rate of $117 \mathrm{cts} \mathrm{s}^{-1}$, the data are considerably affected by coincidence loss, which is however well controlled (Poole et al. 2008; Kuin \& Rosen 2008). The coincidence loss affects the standard deviation during the individual orbits, which is a factor 1.5 higher than the photon noise.

During each visit, we detect variations with a standard deviation of $0.42 \%$ of the average source rates from orbit to orbit. This instrumental error is likely caused by small-scale sensitivity variations of the detector and thus depends on the source position on the detector. The source position is found to be random in the vicinity of the aimpoint after the two correction slews, and therefore this instrumental error can be assumed to be random. Thus, Swift UVOT achieves an accuracy in relative flux measurements of about $0.42 \%$ over several orbits and is suited to measure enhanced NUV transit depths of around 3\% as observed in WASP-12 b. However, multiple in- and out-of-transit exposures are required to achieve sufficient accuracy, which usually requires transit observations to be repeated in several visits.

\section{Transit analysis on the example of $55 \mathrm{Cnc}$}

We analyzed the 55 Cnce data implementing the analytical transit model for a circular orbit from Mandel \& Agol (2002). The model was explored with a Markov chain Monte Carlo (MCMC) method following the emcee implementation (Foreman-Mackey et al. 2013) and the PyAstronomy package ${ }^{2}$. The chain was run

\footnotetext{
2 https://github.com/sczesla/PyAstronomy
} 

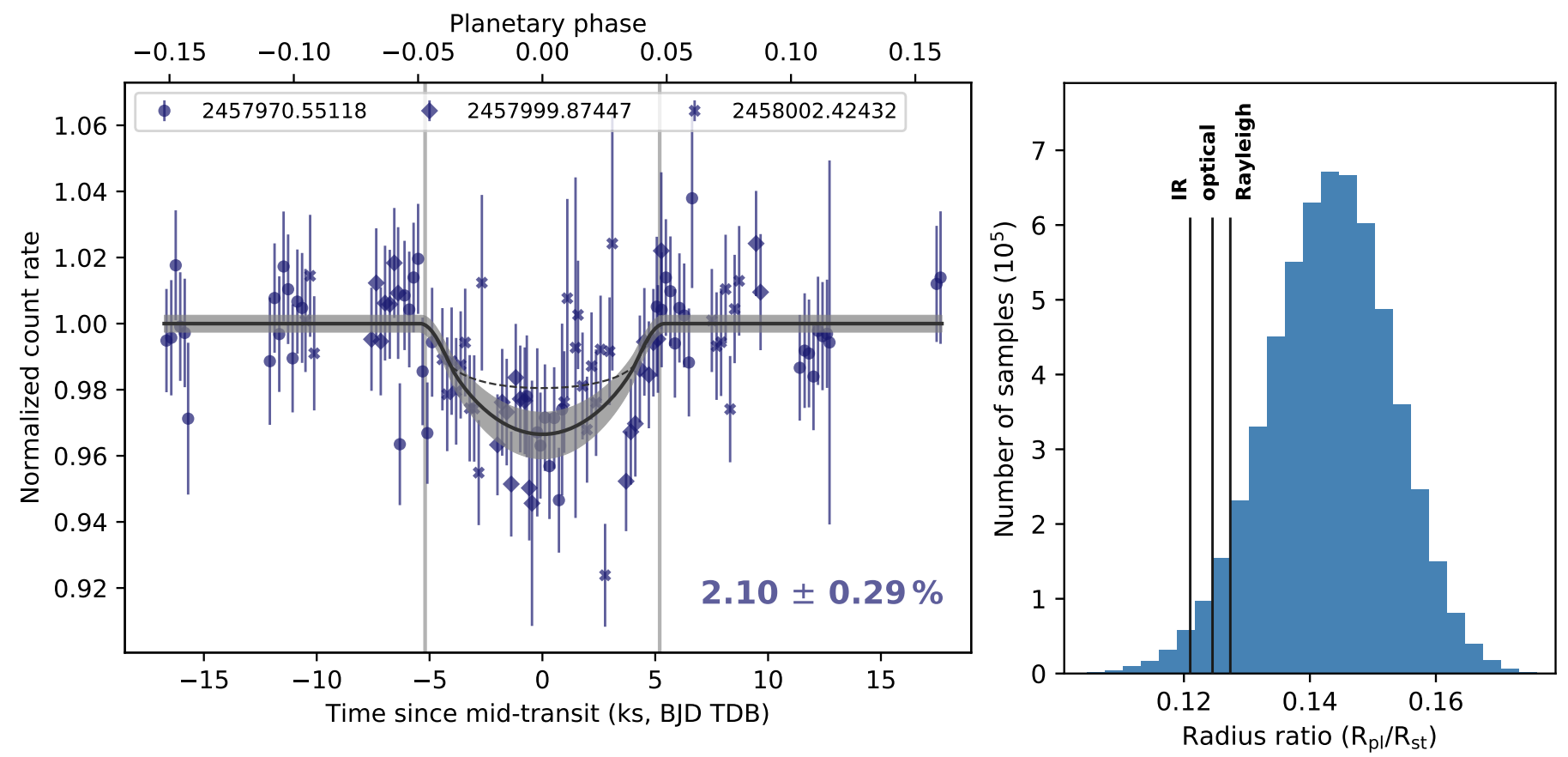

Fig. 2. Left panel: phased light curves of WASP-121 during three transits observed with Swift UVOT labeled by their mid-transit times in BJD. The data are rebinned to $200 \mathrm{~s}$ intervals and corrected for the modeled systematic offsets. The uncorrected data are shown in Fig. A.1. The transit model with $1 \sigma$ uncertainty interval of the out-of-transit flux level and transit depth is shown. The optical transit depth is depicted by the dashed line ( $B$-filter, Delrez et al. 2016). The observed transit depth is given at the bottom with $1 \sigma$ error. Right panel: posterior of the radius ratio. Optical and IR values are indicated (Delrez et al. 2016; Evans et al. 2016) together with the expected NUV transit depth according to Rayleigh scattering.

with the number of independent walkers set to two times the number of free parameters. Each walker had a burn-in of $2 \times$ $10^{4}$ steps and was then propagated over $8 \times 10^{4}$ steps, resulting in a total of $5.9 \times 10^{6}$ samples. Besides the radius ratio between the planet and the star, the model has four parameters (semimajor axis, inclination, orbital period, and reference mid-transit time), which are set up according to the values of Demory et al. (2016). Additionally, we fit the out-of-transit flux level of the host star in each visit individually.

The instrumental error of the UVOT described in the previous section is introduced into the transit model by allowing multiplicative offsets for each individual exposure with normal priors centered at 1.0 and with a standard deviation of 0.0042 . With this procedure, the instrumental error directly affects the marginalized radius ratio and the significance with which a transit is detected.

The data only provide an upper limit for the NUV transit depth of $0.4 \%$ ( $95 \%$ confidence), compared to the optical transit depth of $0.04 \%$.

\section{Ultraviolet transit photometry of WASP-121 b}

Having validated the capabilities of Swift UVOT, we observed three transits of WASP-121 b on Aug. 4, Sept. 3, and Sept. 5, 2017 (observation IDs 00010206001 to 00010206027 ). As for $55 \mathrm{Cnc}$, the $u v m 2$ filter was chosen to avoid the red-leaks present in the other UV filters (Breeveld et al. 2011). The observed flux is dominated by the $200-270 \mathrm{~nm}$ range, which covers an approximately five times broader wavelength range than the HST COS observations of WASP-12 (see Fig. 4). Our first visit of WASP-121 extended over seven spacecraft orbits $(\approx 35 \mathrm{ks})$, which allows us to study pre- and post-transit variability; the following two visits extended over four spacecraft orbits. The data were acquired and reduced as described for $55 \mathrm{Cnc}$ in Sect. 3. We identified and excluded five exposures, which were affected by low-sensitivity patches (see labels 1-5 in Fig. A.1 and the discussion in the appendix.

The transit of WASP-121 b was analyzed following the description in Sect. 4. System parameters were set up with Gaussian priors according to the values and errors given by Delrez et al. (2016) and Evans et al. (2016). These parameters were not further constrained by the UVOT data, but were included as nuisance parameters for their impact on the uncertainty of the transit depth. In total, the model has 37 free parameters: 5 from the transit model, 3 from the out-of-transit flux levels of the three visits, and 29 from the offset parameters for the individual exposures.

The data quality is not sufficient to constrain the coefficients of limb-darkening laws, and for UV filters, they are not available in the literature (e.g., Claret \& Bloemen 2011). Therefore, we used stellar model atmospheres and angle-dependent specific intensities computed with the PHOENIX code (Husser et al. 2013) to derive a theoretical limb-darkening curve for the uvm 2 filter. We chose stellar parameters that match those of WASP-121 but varying the effective temperature in the range $T_{\text {eff }}=6500 \pm 200 \mathrm{~K}$ and the surface gravity in the range $\log _{10} g\left(\mathrm{~cm} \mathrm{~s}^{-1}\right)=4.0-4.5$. The quadratic limb-darkening law as used by Claret \& Bloemen (2011) with the coefficients $a=1.42 \pm 0.08$ and $b=-0.49 \pm 0.08$ fits the theoretical curves of WASP-121-like stars well ${ }^{3}$. We used the best fit limb-darkening coefficients for the MCMC, but checked that varying the limbdarkening coefficients within twice their derived uncertainty margin has a small impact of less than $0.1 \%$ points on the resulting transit depth.

Figure 2 shows the NUV light curves phased to the transit of WASP-121 b. The mean out-of-transit count rate of $35.1 \mathrm{cts} \mathrm{s}^{-1}$

3 We followed the rescaling of the $\mu$-axis as done by Müller (2015). 
Table 1. MCMC best-fit statistics with $1 \sigma$ error in brackets.

\begin{tabular}{lcc}
\hline \hline & Full model & Fixed radius ratio \\
\hline Radius ratio & $0.145(10)$ & $0.12454(48)$ \\
Free parameters & 37 & 36 \\
DOF $^{a}$ & 965 & 966 \\
$\chi_{\text {red }}$ & 1.062 & 1.064 \\
\hline
\end{tabular}

Notes. ${ }^{(a)}$ Degrees of freedom.

varies by nearly $1 \%$ between our visits, indicating slight stellar variability over the observing campaign of one month. The planetary transit is clearly detected with a mean transit depth of $2.10 \pm 0.29 \%$, which is 1.4 times deeper than the optical value of $1.551 \pm 0.012 \%$ measured at around $500 \mathrm{~nm}$, but only at $1.9 \sigma$ level. Rayleigh scattering in a clear atmosphere increases the NUV transit depth at $225 \mathrm{~nm}$ to only $1.62 \%$ (using Eq. (1) of Lecavelier Des Etangs et al. 2008). The right-hand side of Fig. 2 compares the marginalized radius ratio of our MCMC run with the optical and infrared values. In contrast to the WASP-12 system (Fossati et al. 2010; Nichols et al. 2015), we do not find significant indications for pre-transit absorption in the WASP-121 data.

Our analysis indicates excess NUV absorption, but the significance remains low. As a reference, we repeated the MCMC transit analysis keeping the radius ratio fixed to the optical value. Unsurprisingly, this model also yields a satisfactory fit to the data (see Fig. A.1 and Table 1). We find that two factors impede a more significant result: uncertainties in the offsets and the out-of-transit flux levels. In the fixed-radius-ratio model, the in-transit offsets are on average $0.21 \%$ points lower than the outof-transit ones, thus, absorbing about half of the possible excess absorption. It remains unclear whether this is a chance finding, given the relatively small shift of the eight in-transit exposures compared to the assumed standard deviation of $0.42 \%$. Additionally, the out-of-transit flux levels for two visits remain more weakly constrained than the in-transit levels. The fixed-radiusratio model yields estimates for the out-of-transit flux levels on average $0.26 \%$ lower than our standard model (see Fig. A.1), again, neutralizing a potentially deeper transit. Therefore, we can neither rule out nor confirm a physical origin of the deeper transit with certainty.

\section{X-ray emission from WASP-121}

The Swift space observatory provides one advantage compared to HST observations: the X-ray Telescope (XRT, Burrows et al. 2005) operates contemporaneously to the UVOT and can provide the planetary high-energy irradiation level at the time of the transit observation. In our observations, WASP-121 is detected in the first visit with $13.0 \pm 3.9$ source counts $\left(1.26 \pm 0.37 \mathrm{cts} \mathrm{ks}^{-1}\right)$, but the second and third visits result in a nondetection with an upper limit of $0.3 \mathrm{cts} \mathrm{ks}^{-1}$ (95\% confidence). Half of the photons in the first visit are detected in the first spacecraft-orbit, indicating flaring activity at the beginning of the observation. Therefore, WASP-121 shows X-ray variability.

We obtained a source spectrum for the first visit from the online interface of the UK Swift Science Data Centre at the University of Leicester (based on HEASOFT v6.20, Evans et al. 2009). The data were modeled with Xspec (v12.9.1) with an optically thin plasma emission model (APEC, AtomDB v3.0.7, Foster et al. 2012) implementing solar abundances

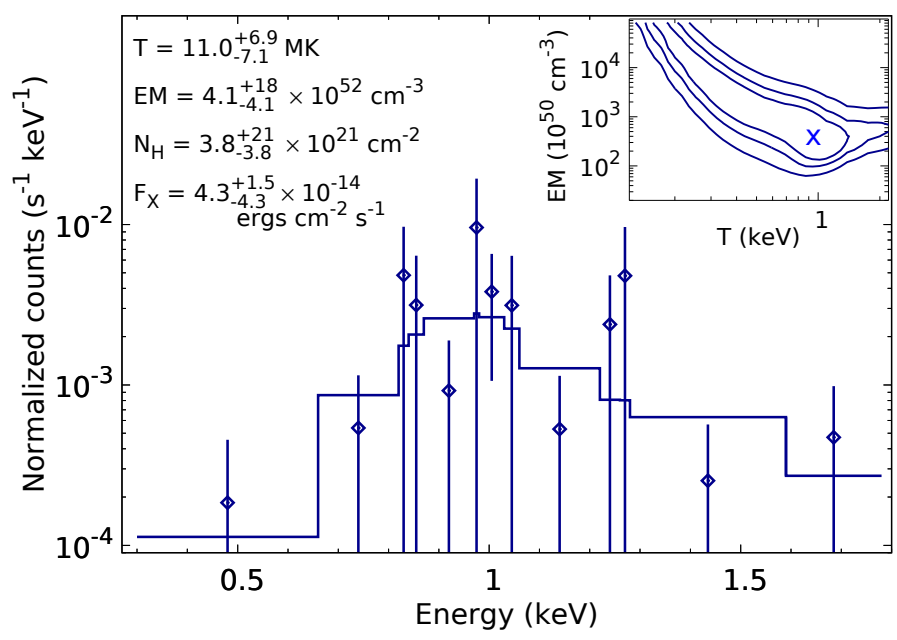

Fig. 3. XRT data of the first visit binned to one count per bin. The depicted model is a one-temperature fit with an interstellar absorption component. The insert shows the $\chi^{2}$ contours of the temperature vs. emission measure, derived with Xspec's steppar function. The fitted model parameters and the resulting flux are provided with $1 \sigma$ uncertainty ranges.

(Grevesse \& Sauval 1998). We included an absorption component via the Tübingen-Boulder ISM absorption model to fit the equivalent hydrogen column (Wilms et al. 2000). The spectrum of WASP-121 shows hot plasma emission with a peak around $1 \mathrm{keV}$; the model parameters with $90 \%$ confidence intervals are given in Fig. 3. Given the distance of $272 \pm 1.6$ pc (Gaia Collaboration 2016, 2018), we find an average X-ray luminosity in the first visit of $L_{\mathrm{X}}(0.2-2.4 \mathrm{keV})=$ $1.0_{-1.0}^{+3.5} \times 10^{30} \mathrm{erg} \mathrm{s}^{-1}$.

For an F-type star with a rotation period of around $1 \mathrm{~d}$, we expect saturated X-ray emission on the order of $10^{30} \mathrm{erg} \mathrm{s}^{-1}$ (Pizzolato et al. 2003), which is only seen during the supposed flare. The nondetection during visits 2 and 3 implies a more than four times lower average X-ray luminosity or a high column density on the order of $10^{21} \mathrm{~cm}^{-2}$ that absorbs an intrinsic X-ray luminous source. Such a high column density is consistent with the data of the first visit, where few soft X-rays are detected. This may indicate an elevated level of system intrinsic absorption caused by material that has been evaporated from the planet as suggested for WASP-12 (Fossati et al. 2013).

\section{Putting WASP-121 and WASP-12 in context}

At face value, the NUV absorption of WASP-121 b is on a par with what has been observed by HST in the WASP-12 system, demonstrating that such extreme systems may commonly exhibit strong excess broadband NUV absorption during transit. The planet's Roche lobe cross-section projected onto the star covers $2.5 \%$ of the stellar disk compared to the $2.1 \%$ NUV transit depth. If confirmed, this suggests that the excess NUV absorption occurs in a dense escaping atmosphere that fills the Roche lobe.

WASP-121 is a fast rotator with a $1 \mathrm{~d}$ period and is presumably more active than WASP-12 with a rotation period estimate of $6 \mathrm{~d}$ (Albrecht et al. 2012). These periods provide canonical estimates for the stellar X-ray luminosities of $\log _{10} L_{\mathrm{X}}\left(\mathrm{erg} \mathrm{s}^{-1}\right)=30.0$ and 29.1 for WASP-121 and WASP-12 (Pizzolato et al. 2003). Based on the X-ray-EUV flux relations from King et al. (2018), we derive irradiation levels 


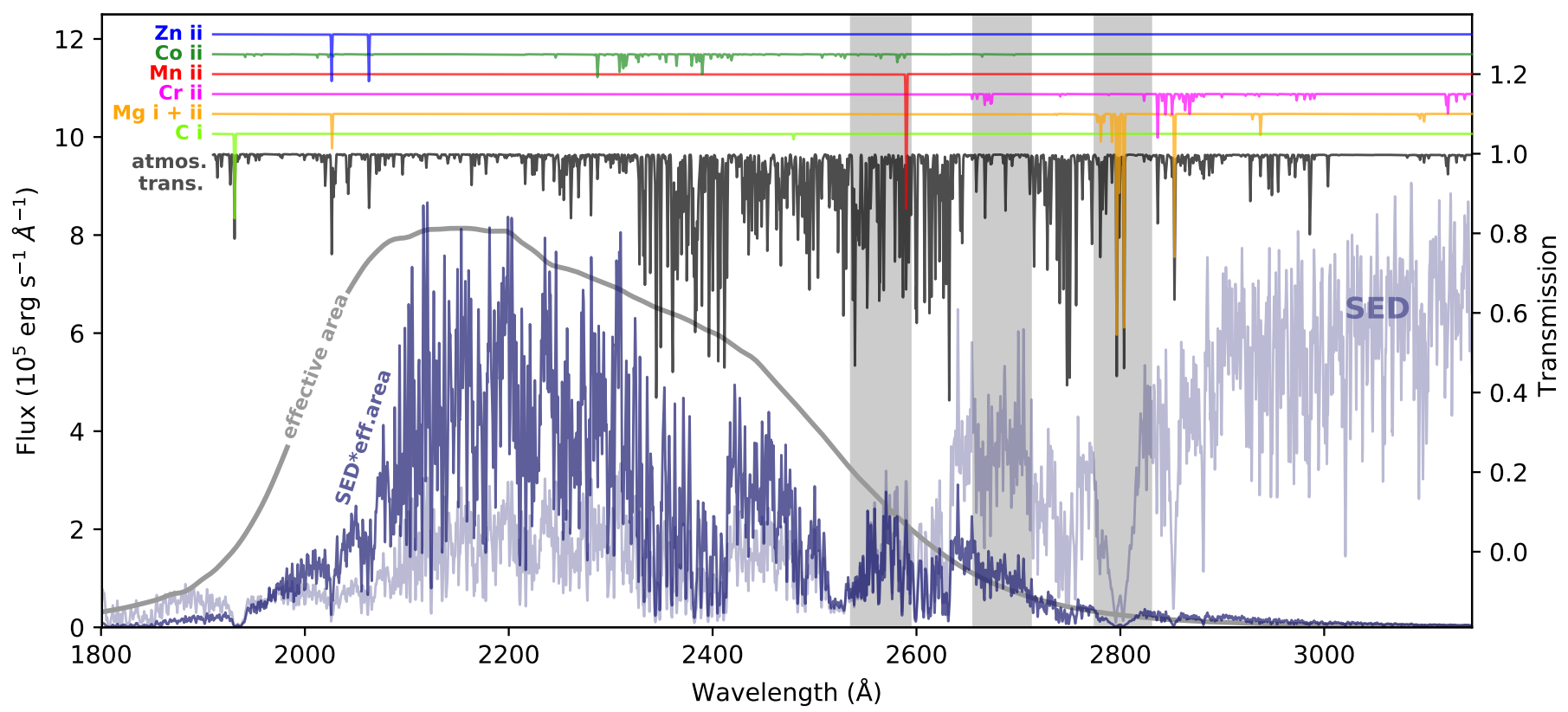

Fig. 4. Model of the SED of WASP-121 at the distance of the planet (light blue). The effective area of the instrument-filter combination and its multiplication with the stellar spectrum are depicted in arbitrary units. An atmospheric transmission model is depicted in black and contributions of individual ions are shown above - all remaining lines are caused by Fe II. Ranges covered by HST COS observations of WASP-12 are shaded. All spectra are shown at a resolution of 3000, the nominal resolution of the CUTE cubesat (Fleming et al. 2018).

of $\log _{10} F_{\mathrm{XUV}}\left(\mathrm{erg} \mathrm{cm}^{-2} \mathrm{~s}^{-1}\right)=6.2$ and 5.6, which results in energy-limited mass-loss rates of $\log _{10} \dot{M}\left(\mathrm{~g} \mathrm{~s}^{-1}\right)=13.7$ and 13.1 for WASP-121 b and WASP- $12 \mathrm{~b}^{4}$ respectively (using the heating efficiency and XUV absorption radius from Salz et al. 2016a).

Although some uncertainties remain, WASP-121 b and WASP-12 b likely experience similarly extreme mass loss on the order of $10^{13} \mathrm{~g} \mathrm{~s}^{-1}$. At much lower mass-loss rates on the order of $\approx 10^{10} \mathrm{~g} \mathrm{~s}^{-1}$, HD $209458 \mathrm{~b}$ and WASP-80 b do not exhibit significantly increased broadband NUV transit depths (Vidal-Madjar et al. 2013; King et al. 2018). Broadband NUV absorption could thus serve as a proxy for strong planetary mass loss. This is also supported by simulations, which show that higher mass loss results in higher thermospheric densities (e.g., Salz et al. 2016b).

\section{Can metals cause broadband NUV absorption?}

Fossati et al. (2010) and Haswell et al. (2012) have provided evidence that the broadband NUV absorption in WASP-12 b is caused by absorption lines of lowly ionized metals. Our observations do not contain spectral information, and the $u v m 2$ filter covers a broader wavelength range than the COS orders. We ran a simulation with the Cloudy code (C17.00, Ferland et al. 2017) to check whether metals in an extended planetary atmosphere can possibly cause the observed absorption level. Cloudy selfconsistently solves the radiative transfer in an irradiated cloud including the 30 lightest elements. We note that the recently updated version of Cloudy was used, which incorporates a major revision of the atomic database with more transitions. We included all available atomic levels for low-ionized metals, the large model atoms for the hydrogen and helium iso-sequences, and neglected molecules.

We setup a gas cloud with a line-of-sight extent of $3 R_{\mathrm{p}}$ and use conditions that can exist in the thermosphere of a strongly

\footnotetext{
4 The derived mass loss of WASP-12 $b$ is higher than literature values (Ehrenreich \& Désert 2011; Salz et al. 2016b), because we assume faster stellar rotation.
}

evaporating hot Jupiter: a uniform gas cloud with solar metallicity, with a temperature of $10^{4} \mathrm{~K}$, and a density of $10^{10} \mathrm{~cm}^{-3}$, leading to a column density of $10^{20.6} \mathrm{~cm}^{-2}$. For the irradiation of the gas, we compute the spectral energy distribution (SED) of WASP-121 with the stellar parameters of Delrez et al. (2016) using the atmosphere code from Shulyak et al. (2004). The computed stellar SED is supplemented by XUV radiation with the reconstructed irradiation level following Salz et al. (2016b). Figure 4 shows the stellar SED and the observed flux in the $u v m 2$ filter.

The simulation exhibits many Fe II absorption lines densely distributed over the spectral range covered by the $u v m 2$ filter (see Fig. 4). A few mostly singly ionized metals contribute to the absorption, most notably the Mg II lines around $2800 \AA$, which were detected in WASP-12 b (Haswell et al. 2012). The Mg I line at $2852 \AA$ was not covered by the WASP-12 data, but was seen in absorption in HD 209458 b (Vidal-Madjar et al. 2013). The central order of the COS data provided the least evidence for excess absorption by WASP-12 b, which is reproduced by our simulation. Relatively strong broadband absorption extends up to about $3000 \AA$, at which point absorption lines become more sparse. With the SED of WASP-121 in the $u v m 2$ filter, we find $0.5 \%$ broadband absorption from this model if $15 \%$ of the stellar disk is covered by the cloud. Without the Fe II ion the absorption level is a factor ten weaker. The computed absorption level is on a par with what is suggested by the observations, showing that Fe II may be responsible for broadband NUV absorption.

\section{Conclusions}

With an archival transit campaign of $55 \mathrm{Cnce}$, we demonstrate that Swift UVOT achieves an accuracy of about $0.5 \%$ for relative flux measurements, which is sufficient for transit observations of hot Jupiters. With three transit observations of WASP-121 b, we detect the planetary transit in the NUV. Our analysis suggests that the broadband NUV transit depth of the hot Jupiter is 
$0.55 \%$ points deeper than in the optical, but the significance of this result remains low $(1.9 \sigma)$. Uncertainties in the out-of-transit level and the instrumental error of the UVOT photometry limit the accuracy with which the NUV transit depth can be measured.

Our Cloudy simulations show that broadband NUV absorption can be caused by Fe II in an extended atmosphere. Similar excess NUV absorption is only known in the WASP-12 system. Planets with one thousand times lower mass-loss estimates than WASP-121 b and WASP-12 b do not exhibit broadband NUV absorption, providing evidence that such absorption may indeed by linked to the extreme planetary mass loss. Future NUV transit-depth measurements provide the opportunity to undertake systematic studies of escaping planetary atmospheres and to test theoretical predictions like the energy-limited nature of planetary mass loss. With a planned mission start in 2020, the CUTE cubesat will address this subject with spectrally resolved NUV transit light curves of short-period planets orbiting bright stars (Fleming et al. 2018).

Acknowledgements. We thank Paul Kuin from the Swift UVOT team for his help regarding the UVOT data reduction. We would also like to thank the second referee for the insightful comments. This work made use of data supplied by the UK Swift Science Data Centre at the University of Leicester. This work has made use of data from the European Space Agency (ESA) mission Gaia (https: //www. cosmos.esa.int/gaia), processed by the Gaia Data Processing and Analysis Consortium (DPAC, https://www. cosmos.esa.int/web/ gaia/dpac/consortium). Funding for the DPAC has been provided by nationa institutions, in particular the institutions participating in the Gaia Multilateral Agreement. M.S. acknowledges support by the DFG SCHM 1032/57-1 and DLR 50OR1710. P.C.S. acknowledges support by DLR 50OR1706.

\section{References}

Albrecht, S., Winn, J. N., Johnson, J. A., et al. 2012, ApJ, 757, 18

Breeveld, A. A., Curran, P. A., Hoversten, E. A., et al. 2010, MNRAS, 406, 1687 Breeveld, A. A., Landsman, W., Holland, S. T., et al. 2011, AIP Conf. Ser., 1358, 373

Burrows, D. N., Hill, J. E., Nousek, J. A., et al. 2005, Space Sci. Rev., 120, 165

Charbonneau, D., Brown, T. M., Latham, D. W., \& Mayor, M. 2000, ApJ, 529, L45

Claret, A., \& Bloemen, S. 2011, A\&A, 529, A75

Delrez, L., Santerne, A., Almenara, J.-M., et al. 2016, MNRAS, 458, 4025

Demory, B.-O., Gillon, M., de Wit, J., et al. 2016, Nature, 532, 207

Ehrenreich, D., \& Désert, J.-M. 2011, A\&A, 529, A136

Ehrenreich, D., Bourrier, V., Wheatley, P. J., et al. 2015, Nature, 522, 459

Erkaev, N. V., Kulikov, Y. N., Lammer, H., et al. 2007, A\&A, 472, 329

Evans, P. A., Beardmore, A. P., Page, K. L., et al. 2009, MNRAS, 397, 1177

Evans, T. M., Sing, D. K., Wakeford, H. R., et al. 2016, ApJ, 822, L4

Evans, T. M., Sing, D. K., Kataria, T., et al. 2017, Nature, 548, 58
Ferland, G. J., Chatzikos, M., Guzmán, F., et al. 2017, Rev. Mex. Astron. Astrofis., 53, 385

Fleming, B. T., France, K., Nell, N., et al. 2018, J. Astron. Telesc. Instrum. Syst., 4, 014004

Foreman-Mackey, D., Hogg, D. W., Lang, D., \& Goodman, J. 2013, PASP, 125, 306

Fossati, L., Haswell, C. A., Froning, C. S., et al. 2010, ApJ, 714, L222

Fossati, L., Ayres, T. R., Haswell, C. A., et al. 2013, ApJ, 766, L20

Foster, A. R., Ji, L., Smith, R. K., \& Brickhouse, N. S. 2012, ApJ, 756, 128

Fulton, B. J., Petigura, E. A., Howard, A. W., et al. 2017, AJ, 154, 109

Gaia Collaboration (Prusti, T., et al.) 2016, A\&A, 595, A1

Gaia Collaboration (Brown, A. G. A., et al.) 2018, A\&A, 616, A1

Grevesse, N., \& Sauval, A. J. 1998, Space Sci. Rev., 85, 161

Haswell, C. A., Fossati, L., Ayres, T., et al. 2012, ApJ, 760, 79

Hebb, L., Collier-Cameron, A., Loeillet, B., et al. 2009, ApJ, 693, 1920

Henry, G. W., Marcy, G. W., Butler, R. P., \& Vogt, S. S. 2000, ApJ, 529, L41

Husser, T.-O., Wende-von Berg, S., Dreizler, S., et al. 2013, A\&A, 553, A6

Khodachenko, M. L., Shaikhislamov, I. F., Lammer, H., \& Prokopov, P. A. 2015, ApJ, 813, 50

King, G. W., Wheatley, P. J., Salz, M., et al. 2018, MNRAS, 478, 1193

Kuin, N. P. M., \& Rosen, S. R. 2008, MNRAS, 383, 383

Kulow, J. R., France, K., Linsky, J., \& Loyd, R. O. P. 2014, ApJ, 786, 132

Lavie, B., Ehrenreich, D., Bourrier, V., et al. 2017, A\&A, 605, L7

Lecavelier Des Etangs, A., Vidal-Madjar, A., Désert, J.-M., \& Sing, D. 2008, A\&A, 485, 865

Lecavelier des Etangs, A., Ehrenreich, D., Vidal-Madjar, A., et al. 2010, A\&A, 514, A72

Lecavelier des Etangs, A., Bourrier, V., Wheatley, P. J., et al. 2012, A\&A, 543, L4

Linsky, J. L., Yang, H., France, K., et al. 2010, ApJ, 717, 1291

Lundkvist, M. S., Kjeldsen, H., Albrecht, S., et al. 2016, Nat. Commun., 7, 11201

Mandel, K., \& Agol, E. 2002, ApJ, 580, L171

Müller, H. M. 2015, Ph.D. Thesis, Universität Hamburg, Hamburg

Murray-Clay, R. A., Chiang, E. I., \& Murray, N. 2009, ApJ, 693, 23

Nichols, J. D., Wynn, G. A., Goad, M., et al. 2015, ApJ, 803, 9

Owen, J. E., \& Alvarez, M. A. 2016, ApJ, 816, 34

Parker, E. N. 1958, ApJ, 128, 664

Pizzolato, N., Maggio, A., Micela, G., Sciortino, S., \& Ventura, P. 2003, A\&A, 397, 147

Poole, T. S., Breeveld, A. A., Page, M. J., et al. 2008, MNRAS, 383, 627

Roming, P. W. A., Kennedy, T. E., Mason, K. O., et al. 2005, Space Sci. Rev., 120,95

Salz, M., Schneider, P. C., Czesla, S., \& Schmitt, J. H. M. M. 2016a, A\&A, 585, L2

Salz, M., Czesla, S., Schneider, P. C., \& Schmitt, J. H. M. M. 2016b, A\&A, 586, A75

Shulyak, D., Tsymbal, V., Ryabchikova, T., Stütz, C., \& Weiss, W. W. 2004, A\&A, 428, 993

Tsiaras, A., Waldmann, I. P., Zingales, T., et al. 2018, AJ, 155, 156

Vidal-Madjar, A., Lecavelier des Etangs, A., Désert, J.-M., et al. 2003, Nature, 422, 143

Vidal-Madjar, A., Huitson, C. M., Bourrier, V., et al. 2013, A\&A, 560, A54

Watson, A. J., Donahue, T. M., \& Walker, J. C. G. 1981, Icarus, 48, 150

Wilms, J., Allen, A., \& McCray, R. 2000, ApJ, 542, 914 


\section{Appendix A: Low-sensitivity patches of UVOT}

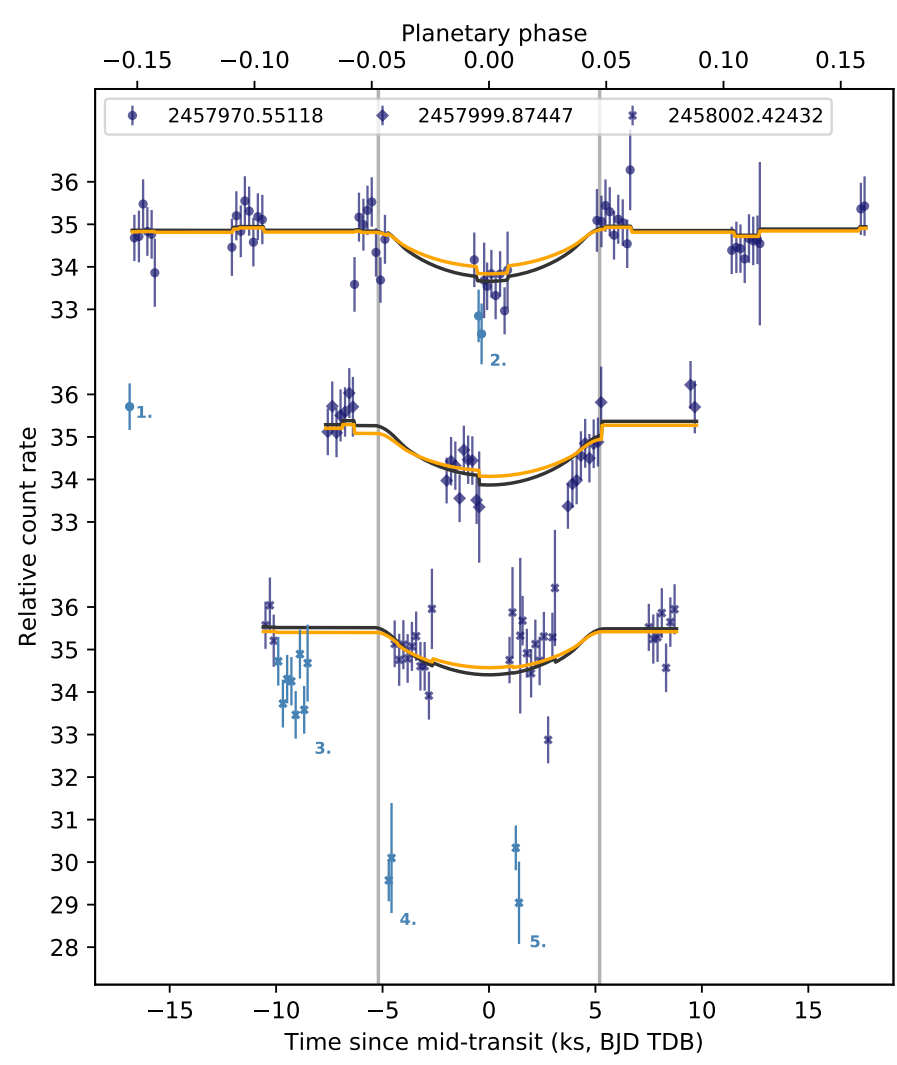

Fig. A.1. Transit light curves of WASP-121 with our model including the offsets caused by instrument systematics (black curve). A model with the transit depth fixed to the broadband optical value is shown by the orange line. Exposures discarded due to low-sensitivity patches are indicated; see labels $1-5$.

The UVOT has known patches of low sensitivity. Usually, a double-correction-slew places the source on clean detector regions for the long science exposure. After the first slew onto the target, the spacecraft retains considerable motion over the first acquisition exposure. This causes the distorted PSF during exposures 1 and 4 in Fig. A.2. A correction slew is then performed, after which the target usually falls within 50 pixels of the aimpoint. The second acquisition exposure is obtained, and after another correction slew, we find the target within 18 pixels of the aimpoint. Continuous monitoring for the rest of the visibility window follows (science exposure).

We identify and exclude four acquisition and one science exposure that are affected by low sensitivity patches (see

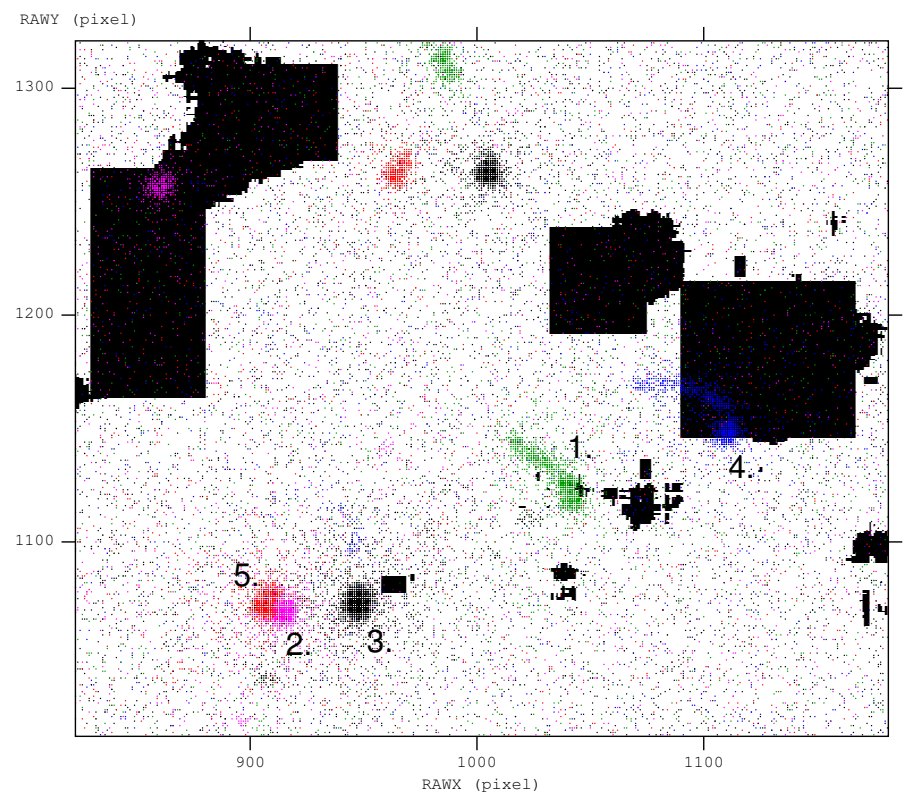

Fig. A.2. Source positions in raw detector coordinates during exposures affected by low-sensitivity patches. The figure shows a central region of the UVOT; known bad patches are shaded. The source positions are numbered according to Fig. A.1. The aimpoint was close to the $X Y$ position 935 and 1060 in raw detector coordinates.

Figs. A.1 and A.2). 1 and 4: in visit 1 - orbit 1 and visit 3 - orbit 2 the source position in the first acquisition exposure coincides with known low-sensitivity patches. 3: during visit 3 - orbit 1 the source position in the science exposure touches a lowsensitivity patch and the source flux is diminished by about $4 \%$ compared to the acquisition exposures in the same orbit. 5: in visit 3 - orbit 3 the second acquisition exposure shows a clearly diminished flux compared with the first and third exposures. There is no known low-sensitivity patch at the source position of $(907,1073)$ in raw detector coordinates. It is unlikely that the host star shows a $15 \%$ drop in flux that happens simultaneously with position changes on the detector. Therefore, we identify a previously unknown patch at this position. 2: we checked all exposures again for the new patch location, and indeed in the second exposure of visit 1 - orbit 4 the stellar PSF partially overlaps with this position. The source flux is also reduced by about 5\% compared with exposures 1 and 3, which supports our identification of a new low-sensitivity patch. These five exposures were excluded from our transit analysis. 